\title{
A reevaluation of angle-contingent color aftereffects
}

\author{
C. A. G. HAYMAN and LORRAINE G. ALLAN \\ McMaster University, Hamilton, Ontario L8S 4K1, Canada
}

\begin{abstract}
It has been argued that adaptation to a series of angles with vertices pointing up and illuminated in one color, and to angles with vertices pointing down and illuminated in the opponent color, results in color aftereffects that are contingent on angle direction. In the present paper, using a number of test figures, we demonstrate that adaptation to these ascending/descending angles results in color aftereffects that can be accounted for in terms of spatially localized, orientation-color pairings. In the light of our results, we suggest that previous inferences concerning angle-contingent color aftereffects should be reconsidered.
\end{abstract}

Contingent aftereffects, originally reported by McCollough (1965), are of theoretical interest, not only because of their remarkable persistence over time, but also as a possible methodology for exploring feature-detector models of human visual perception. McCollough (1965) described an orientationcontingent color aftereffect. During a 3-min adaptation period, her subjects inspected two figures that alternated every few seconds. Under one condition, one adaptation figure consisted of black vertical bars illuminated by orange light and the other consisted of black horizontal bars illuminated by blue light. During the test phase, the subjects viewed an achromatic figure, half of which consisted of black vertical bars on a white background and the other half, of black horizontal bars on a white background. The subjects reported colored aftereffects which were contingent on line orientation. For the above condition, the white background of the vertical bars appeared bluish and the white background of the horizontal bars appeared orangish. This orientationcontingent color aftereffect was interpreted by McCollough (1965) as psychophysical evidence for neural units that are both color- and orientationspecific. She argued that the aftereffect could be understood in terms of color adaptation of orientationspecific edge detectors.

Since the original report by McCollough (1965), color aftereffects contingent on movement direction (e.g., Hepler, 1968; Stromeyer \& Mansfield, 1970), on spatial frequency (e.g., Breitmeyer \& Cooper, 1972; Harris, 1970, 1971; Lovegrove \& Over, 1972; Stromeyer, 1972b), on curvature (Riggs, 1973, 1974;

This research was supported by a National Sciences and Engineering Research Council of Canada grant to L.G.A. We would like to thank L. L. Jacoby and H. M. Jenkins for reading and commenting upon an earlier draft of this manuscript. Requests for reprints should be sent to Lorraine G. Allan, Department of Psychology, McMaster University, Hamilton, Ontario L8S 4K1, Canada.
White \& Riggs, 1974), and on angle (White \& Riggs, 1974) have been reported. Color-contingent aftereffects have also been demonstrated. For example, Held and Shattuck (1971) reported a color-contingent tilt aftereffect; Favreau, Emerson, and Corballis (1972) and Mayhew and Anstis (1972) reported a color-contingent movement aftereffect; and Virsu and Haapasalo (1973) reported a color-contingent spatial frequency aftereffect. Thus, over a period of about 15 years, a variety of contingent aftereffects have been demonstrated in which the perception of color is contingent on a specific figural event or in which the perception of a figural event is contingent on a specific color. These varied contingent aftereffects have generated much speculation about the nature of neural feature detectors. Over (1976), Shute (1979), Skowbo, Timney, Gentry, and Morant (1975), Stromeyer (1978), and White (1978) have provided excellent reviews of the contingent-aftereffect literature.

Of particular interest in the present paper is the conclusion reached by White and Riggs (1974) that the color aftereffects, produced by adaptation to figures containing angles pointing in opposite directions, could not be accounted for in terms of color adaptation of orientation-specific edge detectors. They used as their adapting figures pairs of alternating figures, each figure in the pair containing a series of angles with their vertices pointing either up or down. An example of a pair of their adapting figures is shown in Figure la and of their achromatic test figure in Figure 1b. To aid description, a Keyto-Areas is presented in Figure 1c. Of importance to our discussion is the fact that areas 1 and 3 of the test figure contain one orientation of lines and areas 2 and 4 contain the other orientation.

During adaptation in the White and Riggs study, black ascending arrows were presented on a colored background (e.g., magenta) and black descending arrows on a background of a nearly complementary 
(*)

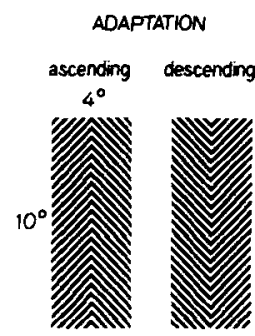

(b)

WHITE \& RIGGS TEST FIGURE

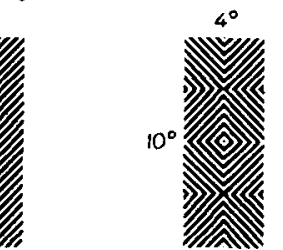

(d) PILOT TEST FIGURE $8^{\circ}$

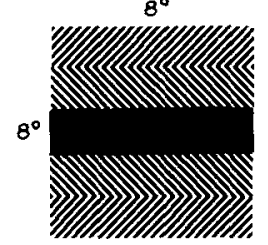

\begin{tabular}{|l|l|}
\hline 3 & 1 \\
\hline 4 & 2 \\
\hline & \\
\hline 4 & 2 \\
\hline 3 & 1 \\
\hline
\end{tabular}

Figure 1. Adaptation figures (10 deg vertical and 4 deg horizontal), the White and Riggs test figure (10 deg vertical and 4 deg horizontal), and the pilot test figure $(8 \mathrm{deg}$ vertical and $8 \mathrm{deg}$ horizontal).

color (green). After adaptation to ascending-magenta/ descending-green, subjects reported that the achromatic test figure was colored by horizontal bands of pink and green: areas 1 and 4 appeared pinkish and areas 2 and 3 appeared greenish. That is, the color aftereffects appeared to be contingent on angle direction and not on line orientation. If the color aftereffects were simply contingent on line orientation, one color would be expected in areas 1 and 3 and the other color in areas 2 and 4. White and Riggs (1974) concluded that "No model based exclusively on line orientation could account for the observed hue aftereffects"' (p. 1148).

Harris (1969), Stromeyer (1972a), and Stromeyer and Dawson (1978) have shown that orientationcontingent color aftereffects are area specific in that they are localized on the area of the retina exposed to the adapting figures. White and Riggs (1974) did consider the possibility that the horizontal color bands perceived on their test figure could be accounted for in terms of spatially localized, orientationcolor pairings during adaptation. With the ascending/ descending arrows as adaptation figures, different orientation-color pairings occur on different parts of the retina. Consider magenta-ascending/greendescending during adaptation. For central fixation, areas to the right of fixation have one set of orientationcolor pairings and areas to the left of fixation have the opposite set of pairings. To be specific, for areas to the right, lines oriented at $45 \mathrm{deg}$ (positive slope) are paired with green and lines oriented at $135 \mathrm{deg}$ (negative slope) are paired with magenta; for areas to the left, lines oriented at $45 \mathrm{deg}$ are paired with magenta and lines oriented at $135 \mathrm{deg}$ are paired with green. Therefore, with the center of the achro-

matic test figure fixated, the aftereffect seen in areas 1 and 4 should be pink and the aftereffect seen in areas 2 and 3 should be green.

White and Riggs (1974) argued that if spatially localized, orientation-color pairings were important, then when the subject shifted his gaze away from the midline of the test figure to the center of one of the areas, that area should be split by the appearance of two opponent colors. Not one of their subjects reported this split-color area for their test figure. "Rather, some subjects occasionally reported that areas 1 and 3 appeared one hue and areas 2 and 4 the other hue" (p. 1149). This aftereffect is not contingent on angle direction, and White and Riggs concluded that "spatially localized line orientation makes a partial contribution" (p. 1149). However, this aftereffect ( 1 and 3 one hue and 2 and 4 the other hue) would not be expected from spatially localized, orientation-color pairings during adaptation if the subject were focusing at the midline of the test area. Rather, it would occur if the subject were fixating at the exterior edge of (or outside) the test figure. It may be that it is difficult to maintain fixation on the unmarked center of the test area. Furthermore, it is reasonable to assume that if some subjects did not fix their gaze at the instructed location and looked instead at the exterior edge of the test figure, it may have been that other subjects looked at the center of the complete test figure. If these subjects did in fact focus at the midline of the test figure, then failure to obtain reports of a split-color area cannot be critical evidence for or against accounts based on angles or on orientation, since both models predict the same aftereffect.

An informal observation led us to reconsider the possibility that line orientation may have been an important determinant of the color aftereffects observed by White and Riggs (1974). Our adaptation figures were identical to one pair used by White and Riggs. The achromatic test figure was the two adaptation figures presented simultaneously, separated by a black vertical band, and rotated through $90 \mathrm{deg}$. This test figure is shown in Figure 1d and a Key-toAreas in Figure 1e. The subject was instructed to fixate the middle of the test figure. For ascendingmagenta/descending-green adaptation figures, all subjects reported that areas 1 and 4 of the test figure appeared pinkish and that areas 2 and 3 appeared greenish. The two diagonally located pink patches and the two diagonally located green patches seen on each arrow of our test figure could not be contingent on angle direction. However, they are consistent with spatially localized, orientation-color pairings during adaptation.

Given the evidence from our pilot work that the ascending/descending adaptation figures may produce orientation-contingent, but highly localized, aftereffects, and given the uncertainty of gaze fixa- 
tion in the White and Riggs study, we decided to explicitly investigate the establishment of spatially localized, orientation-color pairings during adaptation with the White and Riggs figures. We used four different test figures: the White and Riggs figure, an area of the White and Riggs figure presented in isolation (the diagonal-line test figure), the two adaptation figures presented simultaneously (the verticalarrows test figure), and the two adaptation figures rotated $90 \mathrm{deg}$ and presented simultaneously (the horizontal-arrows test figure). The White and Riggs test figure was included to provide baseline data to compare with those of White and Riggs (1974). The other three test figures were constructed to provide data relevant to the establishment of spatially localized, orientation-color pairings during adaptation.

\section{METHOD}

\begin{abstract}
Apparatus
Three standard $35-\mathrm{mm}$ slide projectors were used to present the stimuli-one projector for each of the adaptation figures and one projector for the test figure. The subject was seated $120 \mathrm{~cm}$ from the screen. All figures were created from black and white lines (Letratone No. LT107). The width of each line subtended $10 \mathrm{~min}$ of visual angle. The lines were oriented at either 45 or $135 \mathrm{deg}$. The sizes of the figures, in degrees of visual angle, are indicated in Figures 1 and 2.
\end{abstract}

The adaptation figures were the ascending and descending arrows used by White and Riggs and are shown in Figure 1a. Three of the four types of test figures are diagrammed in Figure 2. The two versions of the diagonal-line test figure (45 and $135 \mathrm{deg}$ ) are presented in Figure 2a. Each version represents an area of the White and Riggs test figure with the addition of a vertical fixation line at the center. The two versions of the vertical-arrows test figure (left-ascending and left-descending) are presented in Figure $2 \mathrm{~b}$. This test figure is composed of the two adaptation figures separated by a black vertical band subtending a visual angle of $1.6 \mathrm{deg}$ horizontally. The two versions of the horizontal-arrows test figure are presented in Figure $2 \mathrm{c}$. This test figure is the vertical-arrows test figure rotated $90 \mathrm{deg}$. The fourth test figure is the one used by White and Riggs and is shown in Figure $1 \mathrm{~b}$.

Each adaptation figure was projected through an Eastman Kodak filter: a Wratten No. 53 filter for green illumination and a Wratten No. 32 filter for magenta illumination.

For two of the test figures, the diagonal-line figure and the vertical-arrows figure, the strength of the color aftereffect was measured with a colorimeter similar to that described by Riggs, White, and Eimas (1974). The test slide was vertically divided and each half was covered with orthogonal polarizing filters. A rotatable disk was mounted in front of the projector lens, at a position corresponding to the focal plane of the projector light source. The disk consisted of a matrix of complementary color filters (CC30G for green and CC20M for magenta), each color filter being covered with orthogonal polarizing filters. The function of the disk was to supply the test figure with a mixture of magenta and green light. The relative amounts of green and magenta on the left and right sides of the test figure can be adjusted by changing the orientation of the polarizing filters on the disk relative to those on the test figure by rotating the disk. At one extreme of rotation, the left side of the test figure would receive only green light and the right side only magenta light; at the other extreme, the left side would receive only magenta light and the right side only green light. In the absence of a color aftereffect, the subject should set the disk so that approximately equal amounts of green and magenta would be present on each side and both sides would appear achromatic. This balance would appear as a
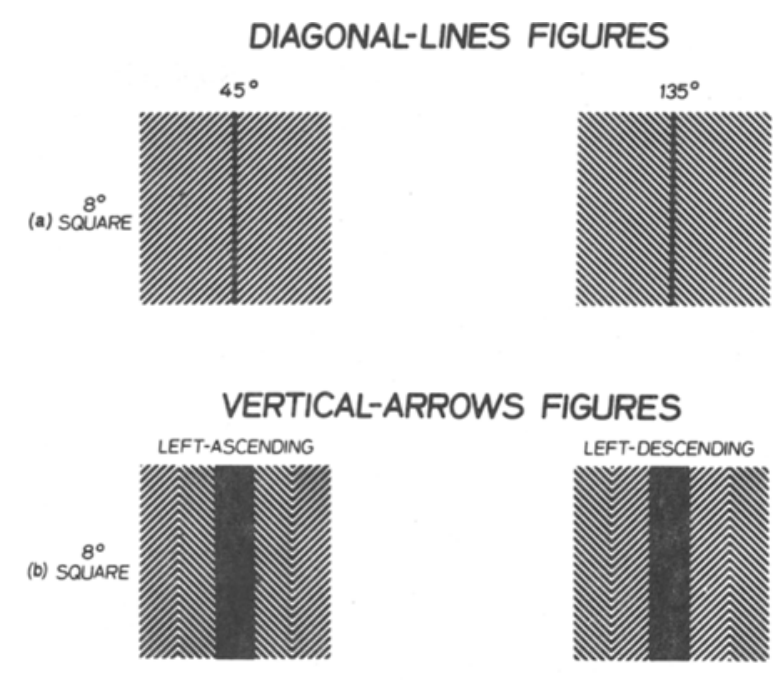

HORIZONTAL-ARROWS FIGURES
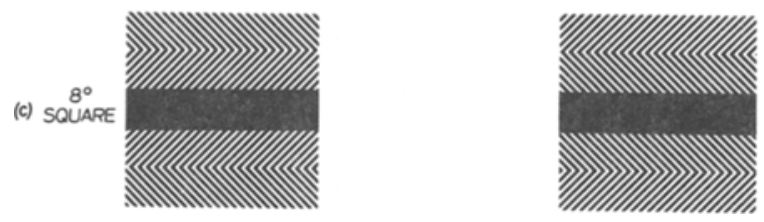

Figure 2. Three of the test figures used in the present experiment: (a) the two versions of the diagonal-line figure, (b) the two versions of the vertical-arrows figure, and (c) the two versions of the horizontal-arrows figure. Each figure subtended a visual angle of $8 \mathrm{deg}$ vertically and $8 \mathrm{deg}$ horizontally.

mismatch if a color aftereffect were present. It would be necessary to add more green to one side and more magenta to the other side in order for an achromatic test figure to be seen. In essence, the colorimeter provides a measure of the amount of color necessary to perceptually counterbalance or nullify a color aftereffect.

\section{Subjects}

Twelve paid subjects participated. Only one of these subjects had previous experience in contingent color aftereffect tasks. Subjects were tested individually.

\section{Procedure}

The experimental session was divided into three phases: baseline, adaptation, and test. The baseline and test phases were conducted under normal room illumination. The adaptation phase occurred in a darkened room under indirect illumination by a 25 -W incandescent bulb.

During the baseline phase, colorimeter readings were obtained from each subject for one version of the diagonal-line test figure and for one version of the vertical-arrows test figure. Each version was used equally often across subjects. Half the subjects received the diagonal-line test figure first and the vertical-arrows test figure second; the order was reversed for the remaining subjects. The method of adjustment was used. The experimenter set the disk so that half of the test figure was clearly magenta and half was clearly green. The subject was instructed to fixate the center of the figure (the vertical line on the diagonal-line figure and the black band on the vertical-arrows figure) and to rotate the disk until the test figure appeared achromatic. Four readings were taken for each test figure. For half of the adjustments, the left side of the figure 
was set at magenta (and the right side at green); for the remaining half of the adjustments, the reverse was the case.

The adaptation period was $15 \mathrm{~min}$. The two adaptation figures were alternated every $5 \mathrm{sec}$. For half of the subjects, the ascending arrows were illuminated by magenta light and the descending arrows by green light; the reverse was the case for the remaining subjects. Fixation was not controlled or monitored.

Two minutes under normal illumination separated the adaptation phase from the test phase. During the test phase, four colorimeter readings were again obtained for each of the two test figures, the order of presentation of the two test figures being the same as during the baseline phase. For the vertical-arrows figure, the subject was told to rotate the disk until the inside (nasal) sections of the two arrows appeared achromatic. The colorimeter settings were followed by verbal color reports about the remaining two test figures-first, one version of the horizontal-arrows figure and then the White and Riggs figure. The subject was asked whether he saw any colors in the test figure, and, if yes, what colors and at what locations. He was then asked to describe what happened with shifts in fixation. Seven of the subjects then gave verbal color reports about one area of the complete White and Riggs figure, about one version of the diagonal-line figure, and about one version of the vertical-arrows figure.

\section{RESULTS AND DISCUSSION}

\section{Colorimeter Readings}

Measuring the strength of the color aftereffect with the colorimeter appears to be a highly reliable technique. The mean baseline setting of the colorimeter across subjects was virtually identical for the two test figures (138.8 deg for the diagonal-line figure and $138.9 \mathrm{deg}$ for the vertical-arrows figure), with a between-subject standard deviation of .85 and $.70 \mathrm{deg}$, and a mean within-subject standard deviation of .80 and $.97 \mathrm{deg}$ for the diagonal-line figure and for the vertical-arrows figure, respectively.

For each subject, the deviation of the mean of the four colorimeter settings during the test phase from the mean of the four settings during baseline was determined. These deviation scores are presented in Table 1 for each test figure. A positive deviation score indicates that for the perception of an achromatic test figure, magenta light was required on the left side of the test figure and green light on the right side; a negative score indicates the reverse.

For the diagonal-line test figure, all 12 subjects showed a nonzero deviation score, and for nine of the subjects the deviation score exceeded 2 deg. The color aftereffect that was observed can be accounted for by spatially localized, orientation-color pairings during adaptation. The magenta-ascending/greendescending adaptation figures should result in a green aftereffect on the left and a pink aftereffect on the right for the 45-deg diagonal-line figure and the reverse for the 135-deg figure. For the 45-deg figure to appear achromatic to the subject, magenta light is required on the left and green on the right (a positive deviation score); for the 135-deg figure to appear achromatic, green light is required on the left and magenta on the right (a negative deviation score). For the other pair of adaptation figures, a negative deviation score is expected for the 45-deg figure and a positive score for the 135-deg figure.

One could argue that there are angles on the diagonal-line test figure, formed by the intersection of the diagonal lines and the vertical fixation line. However, if the observed color aftereffects were contingent on these angles, one would expect the aftereffect to be strongest at the center of the test figure. Verbal reports (discussed in greater detail later) do not support this expectation. The aftereffects are extremely desaturated around the vertical fixation line.

Eleven of the 12 subjects gave deviation scores that exceeded $2 \mathrm{deg}$ on the vertical-arrows test figures. Again, the pattern of results can be accounted for by spatially localized, orientation-color pairings during adaptation. For the left-descending figure, the inside (nasal) line components of each arrow are oriented at 45 deg. Therefore, a positive deviation score should be obtained for the magenta-ascending/ green-descending adaptation figures and a negative score for the other pair of adaptation figures. For the left-ascending figure, the inside (nasal) line components are oriented at $135 \mathrm{deg}$, and a negative score is expected for the magenta-ascending/green-descending adaptation figures and a positive score for the other pair of adaptation figures.

The color aftereffect obtained with the verticalarrows figure is not contingent on angle direction. If the aftereffect were angle-contingent, then, for the magenta-ascending/green-descending adaptation figures, one would expect the descending test arrows to appear pink and the ascending test arrows to ap-

Table 1

Colorimeter Deviation Scores in Degrees for Each Subject on Two Test Figures

\begin{tabular}{|c|c|c|c|c|c|c|c|c|}
\hline \multirow[b]{2}{*}{ Adaptation } & \multirow[b]{2}{*}{ Subject } & \multirow[b]{2}{*}{$\begin{array}{l}\text { Diagonal-Line } \\
45 \mathrm{Deg}\end{array}$} & \multicolumn{2}{|c|}{ Vertical-Arrows } & \multirow[b]{2}{*}{ Subject } & \multirow[b]{2}{*}{$\begin{array}{c}\text { Diagonal-Line } \\
135 \text { Deg }\end{array}$} & \multicolumn{2}{|c|}{ Vertical-Arrows } \\
\hline & & & $\begin{array}{l}\text { Left- } \\
\text { Ascending }\end{array}$ & $\begin{array}{c}\text { Left- } \\
\text { Descending }\end{array}$ & & & $\begin{array}{c}\text { Left- } \\
\text { Ascending }\end{array}$ & $\begin{array}{l}\text { Left- } \\
\text { Descending }\end{array}$ \\
\hline $\begin{array}{l}\text { Magenta-Ascending/ } \\
\text { Green-Descending }\end{array}$ & $\begin{array}{l}\text { P.G. } \\
\text { B.W. } \\
\text { D.S. }\end{array}$ & $\begin{array}{l}2.87 \\
4.00 \\
3.37\end{array}$ & -2.12 & $\begin{array}{r}.12 \\
5.88\end{array}$ & $\begin{array}{l}\text { D.W. } \\
\text { B.Z. } \\
\text { P.B. }\end{array}$ & $\begin{array}{l}-3.13 \\
-1.62 \\
-6.75\end{array}$ & -8.25 & $\begin{array}{l}10.13 \\
11.75\end{array}$ \\
\hline $\begin{array}{l}\text { Magenta-Descending/ } \\
\text { Green-Ascending }\end{array}$ & $\begin{array}{l}\text { P.Y. } \\
\text { M.S. } \\
\text { D.L. }\end{array}$ & $\begin{array}{l}-4.37 \\
-2.75 \\
-2.13\end{array}$ & $\begin{array}{l}6.00 \\
6.50\end{array}$ & -2.87 & $\begin{array}{l}\text { V.A. } \\
\text { B.K. } \\
\text { M.M. }\end{array}$ & $\begin{array}{l}1.00 \\
5.87 \\
1.88\end{array}$ & $\begin{array}{l}3.37 \\
3.25\end{array}$ & -3.62 \\
\hline
\end{tabular}


pear green. Therefore, the deviation score should be positive for the left-ascending test figure and negative for the left-descending figure. This is exactly opposite to the pattern of results shown in Table 1 , as are the angle-contingent predictions for the other pair of adaptation figures.

Our colorimeter data clearly demonstrate that the ascending/descending adaptation figures produce strong consistent color aftereffects that can be explained in terms of spatially localized, orientationcolor pairings during adaptation.

\section{Verbal Reports}

The verbal reports of all 12 subjects about the horizontal-arrows test figure were similar to those of the pilot subjects and consistent with spatially localized, orientation-color pairings during adaptation. Adaptation to magenta-ascending/greendescending resulted in areas 1 and 4 being perceived as pinkish and areas 2 and 3 as green; the reverse was the case for the other pair of adaptation figures. Most subjects reported that the boundaries between the colored areas moved about with eye fixation. That is, if the subject shifted his gaze from the center of the test figure to the right, the color boundary also shifted right. For 8 of the 12 subjects, when instructed to fixate a point outside the test figure, the vertically split color field disappeared. For example, after adaptation to magenta-ascending/ green-descending, if fixation was to the right of the test figure, then all 45-deg sections of the figure appeared green and all 135-deg sections appeared pinkish.

Verbal reports of 10 of the 12 subjects about the White and Riggs test figure, with central fixation, were similar to those reported by White and Riggs (1974)-horizontal bands of opponent colors. (V.A. reported areas 2 and 4 as green and areas 1 and 3 as having no color. B.K. reported that each single test area was vertically split by two colors.) For a single area within the complete White and Riggs figure, the experimenter outlined the appropriate area with a pointer and asked the subject to fixate the center of the area. Of the seven subjects, four reported that the area was vertically split by two opponent colors, in accord with the prediction based on spatially localized, orientation-color pairings during adaptation. For the other three subjects, the test area appeared essentially colorless.

With fixation on the vertical line of the diagonalline figure, four subjects reported that the figure had two colors, one on either side of the vertical fixation line. For the other three subjects, the figure appeared colorless. With fixation on the black band of the vertical-arrows test figure, six subjects reported that each arrow was composed of two colors (the left half of each arrow was seen as one color and the right half as the opponent color), and one subject reported that the test figure had no color. When fixation was shifted to a single arrow, that arrow appeared as a uniform color.

Thus, in general, localized orientation-contingent color aftereffects were reported for all the test figures. Deviant verbal reports were in the direction of no color reports.

A frequent verbal report for each of the test figures when fixated centrally was a relative desaturation of the figure at the vertical midline. This desaturation is not surprising, since fixation was not controlled or monitored during the 15 -min adaptation period. It is likely that the center of the fovea was exposed about equally to each orientation in both colors and therefore that orientation-specific adaptation did not occur.

\section{CONCLUDING COMMENTS}

The reported changes in color on our test figures as a result of shifts in fixation are exactly what one would expect if the color aftereffects were spatially localized. Particularly compelling are the color changes due to shifts in fixation from the black band of the vertical-arrows figure to the center of one of the arrows. With fixation on the black band, each arrow is vertically split by two opponent colors; with fixation on one arrow, that arrow appears as a uniform color. For the horizontal-arrows figure, an equally dramatic perceptual change occurs with a shift in fixation. With central fixation, two diagonally located pink patches and two diagonally located green patches are seen on each arrow. With fixation to one side of the figure, many subjects reported that all 45-deg lines were perceived as one color and all 135-deg lines as the opponent color.

The verbal reports from our subjects about the complete White and Riggs test figure were similar to the reports obtained by White and Riggs from their subjects. As White and Riggs note, these perceived horizontal bands of color could be contingent on either line orientation or angle direction. The fact that not one of their eight subjects reported seeing a split-color area, when asked to fixate the center of one of the areas of the complete figure, led them to infer that the aftereffect was mainly angle contingent. However, four of the seven subjects in the present experiment, who were asked to fixate the center of one area of the complete figure, did report two opponent colors which were appropriately located. It could be that we were more successful than White and Riggs (1974) in obtaining reports of a split-color area because we were very explicit in pointing out where we wanted the subject to focus. It is important to remember that the reports given by some of the White and Riggs subjects, that areas 1 and 3 were of one hue and areas 2 and 4 of the opponent hue, are inconsistent with an account based on angle 
contingency. However, these reports are expected on the basis of localized color aftereffects that are orientation contingent, if it is assumed that the subject focused at the edge of, or outside, the test figure. The data obtained from the White and Riggs subjects, under instructions to fixate the center of a single area, do not provide a strong basis for rejecting an account based on spatially localized, orientationcolor pairings during adaptation.

Our test figures were purposely designed to maximize fixation stability and consistency so that a splitcolor field could be revealed. For all our test figures, both colorimeter settings and verbal reports indicate strong and consistent aftereffects, which can be explained only in terms of spatially localized, orientationcolor pairings during adaptation.

Riggs (1973) and White and Riggs (1974) have reported color aftereffects that appear to be curvature contingent. These aftereffects, along with the apparent angle-contingent color aftereffects, led White and Riggs (1974) to suggest that both aftereffects are mediated by broadly tuned mechanisms that are sensitive to direction of departure from a straight line. Blakemore and Over (1974), Crassini and Over (1975), MacKay and MacKay (1974), Sigel and Nachmias (1975), and Stromeyer (1974) have questioned whether the aftereffects reported by Riggs (1973) and by White and Riggs (1974) are in fact curvature contingent. They report data that indicate that the original curvature results can be accounted for in terms of color adaptation of orientation-specific neural units. Riggs (1974) has raised objections to some of the new data and their interpretation, and Cavill and Robinson (1976) have presented further data which they claim cannot be accounted for in terms of color adaptation of orientation-specific neural units. However, overall, a strong case has been made that curvature-contingent color aftereffects do not necessarily provide psychophysical evidence for neural curvature detectors.

Our data do not rule out the possibility that the ascending/descending adaptation figures produce aftereffects that are partially angle contingent. Rather, they indicate that these figures produce localized color aftereffects that are orientation-contingent and they suggest that some of the inferential bases for angle-contingent aftereffects should be reconsidered. The present findings, in conjunction with the data reported by Blakemore and Over (1974), Crassini and Over (1975), MacKay and MacKay (1974), Sigel and Nachmias (1975), and Stromeyer (1974), indicate that it may not be necessary to postulate a broadly tuned mechanism, sensitive to direction of departure from a straight line, to account for color aftereffects that appear to be contingent on curvature or on angle.

\section{REFERENCES}

Blakemore, C., \& Over, R. Curvature detectors in human vision? Perception, 1974, 3, 3-7.

Breitmeyer, B. G., \& Cooper, L. A. Frequency-specific color adaptation in the human visual system. Perceprion \& Psvchophysics, 1972, 11, 95-96.

Cavill, J., \& Robinson, J. O. A color aftereffect contingent on complex pattern features. Perception \& Psychophysics, 1976, $19,454-459$

Crassini, B., \& Over, R. Curvature-specific color aftereffects. Perception \& Psychophysics, 1975, 17, 398-404.

Favreau, O. E., Emerson, V. F., \& Corballis, M. C. Motion perception: A color-contingent aftereffect. Science, 1972, 176, 78-79.

HARris, C. S. Retinal localization of orientation specific color aftereffects. Journal of the Optical Society of America, $1969,59,504$

HARRIS, C. S. Effect of viewing distance on a color aftereffect specific to spatial frequency. Psychonomic Science, 1970, 21, 350.

Harris, C. S. Orientation-specific color aftereffects dependent on retinal spatial frequency rather than on stripe width. Journal of the Optical Society of America, 1971, 61, 689.

Held, R., \& Shattuck, S. R. Color-and edge-sensitive channels in the human visual system: Tuning for orientation. Science, $1971,174,314-316$.

Heplea, N. Color: A motion-contingent aftereffect. Science, $1968,162,376-377$.

Lovegrove, W. J., \& Over, R. Color adaptation of spatial frequency detectors in the human visual system. Science, 1972. 176, 541-543.

MacKaY, D. M., \& MacKaY, V. Do curvature-contingent chromatic aftereffects require "detectors for curvature"? Vision Research, 1974, 14, 1285-1287.

MAYHEW, J. E. W., \& Anstis, S. M. Movement aftereffects contingent on color, intensity, and pattern. Perception \& Psychophysics, 1972, 12, 77-85.

McCollough, C. Color adaptation of edge-detectors in the human visual system. Science, 1965, 149, 1115-1116.

Over, R. Contingent aftereffects in the perception of colour and contour. In R. H. Day \& G. S. Stanley (Eds.), Studies in perception. Perth: University of Western Australia Press, 1976.

RiGGs, L. A. Curvature as a feature of pattern vision. Science, 1973, 181, 1070-1072.

Riggs, L. A. Curvature detectors in human vision? Science, $1974,184,1200-1201$

RigGs, L. A., White, K. D., \& Eimas, P. D. Establishment and decay of orientation-contingent aftereffects of color. Perception \& Psychophysics, 1974, 16, 535-542.

ShUte, C. C. D. The McCollough effect. Cambridge, England: Cambridge University Press, 1979.

SigEL, C., \& NACHMIAS, J. A re-evaluation of curvature-specific chromatic aftereffects. Vision Research, 1975, 15, 829-836.

Skowbo, D., Timney, B., Gentry, T., \& Morant, R. McCollough effects: Experimental findings and theoretical accounts. Psychological Bulletin, 1975, 82, 497-510.

Stromeyer, C. F., III. Contour-contingent color aftereffects: Retinal area specificity. American Journal of Psychology, 1972, 85, 227-235. (a)

Stromeyer, C. F., III. Edge-contingent color aftereffects: Spatial frequency specificity. Vision Research, 1972, 12, 717-733. (b)

Stromeyer, C. F., III. Curvature detectors in human vision? Science, 1974, 184, 1199-1200.

Stromeyer, C. F., III. Form-color aftereffects in human vision. In R. Held, H. Leibowitz, \& H.-L. Teuber (Eds.), Handbook of sensory physiology (Vol. 8) Perception. Heidelberg, West Germany: Springer-Verlag, 1978. 
Stromeyer, C. F., III, \& Dawson, B. M. Form-colour aftereffects: Selectivity to local luminance contrast. Perception, $1978,7,407-415$.

Stromeyer, C. F., III, \& Mansfield, R. Colored aftereffects produced with moving edges. Perception \& Psychophysics, 1970, 7, 108-114.

Virsu, V., \& HaApasalo, S. Relationships between channels for colour and spatial frequency in human vision. Perception, 1973 , $2,31-40$.
White, K. D. Studies of form-contingent color aftereffects. In J. Armington, J. Kraskopf, \& B. Wooten (Eds.), Visual psychophysics: Its physiological basis. New York: Academic Press, 1978.

White, K. D., \& Riggs, L. A. Angle-contingent color aftereffects. Vision Research, 1974, 14, 1147-1154.

(Received for publication September 20, 1979; revision accepted March 17, 1980.) 